\title{
The Impact of Different Inflow Conditions on the Wake of Horizontal Axis Wind Turbine
}

\author{
\{Ali M. AbdelSalam*, M. R. Abuhegazy ${ }^{\dagger}$, I. M. Sakr and W. A. El-Askary $\}^{\S}$
}

\begin{abstract}
This study introduces a numerical investigation on the impact of different inflow atmospheric conditions on the wind turbine wakes. The effects of the inflow turbulence intensity and wind speed under thermally-stratified atmospheric boundary layer (ABL) are presented and discussed. The steady state three dimensional Reynolds-Averaged Navier-Stokes (RANS) equations are solved in the simulation, along with the Actuator Disk Method (ADM) for the turbine rotor modeling. A modified $k-\varepsilon$ model, namely El Kasmi model, is adopted for the turbulence modulation. Further, an additional source term is added to the turbulence equations, to artificially represent the buoyancy generated turbulence, without the need to solve the energy equation. It is found that, there is a considerable effect of the different atmospheric flow properties on the wake flow behavior. Particularly, as the turbulence intensity increases, the wake recovers faster and hence, the wake deficit decreases and the available wind power in the wake region increases. Further, the wake deficits values immediately downstream the turbine are higher for the lower inflow wind speeds.
\end{abstract}

Keywords: Wind turbine wakes; actuator disk; atmospheric boundary layer; wake deficit; wind power

\section{Introduction}

Wind turbines usually operate in the lowest region of the Atmospheric Boundary Layer (ABL). Accordingly, the evolution and recovery of the wind turbine wakes are expected to be strongly affected not only by the turbine characteristics but also, by complexity of the terrain, the ambient wind speed, and turbulence levels, related to the different atmospheric flow conditions. Therefore, a proper modeling of all these factors becomes mandatory for detailed understanding and accurate prediction of the wind turbine wakes as well as their effects on the performance of the whole wind farm.

Many studies have been conducted on full-scale as well as small-scale wind turbines to investigate the structure of the wakes. Chamorro and Porté-Agel [1] and [2] studied the wake behavior of a model wind turbine, operating in the neutrally-stratified and stably-stratified boundary layer over smooth and rough surfaces. It was elucidated that, the different surface roughness influenced the incoming boundary layer flow characteristics, which in turn affected

\section{Assistant Professor.}

Assistant Lecturer, $\underline{\mathrm{m} \_ \text {abuhegazy91@sh-eng.menofia.edu.eg }}$

Professor.

Dept. of Mech. Power Engineering, Menoufia University, Shebin El-Kom, Egypt. 
the flow structure within the wake region. The velocity deficit and turbulence intensity decreased with downstream distance as the wake expanded. It was also observed that, the negative buoyancy, experienced in the stable regime, produced an enhancement of the turbulence in the lower part of the wake. As a result, the velocity deficit in the near wake region was larger in the neutral case compared with the stable one.

Maeda et al. [3] investigated experimentally the effect of the turbulence intensity of the external flow on the wake structure, generated behind a wind turbine. They concluded that, the wake is recovered faster as the turbulence intensity increases. Further, the locations of the maximum turbulence intensity values were found to be near the blade tips and not affected by any inflow conditions.

Zhang et al. [4] studied the effects of the thermally-stratified Convective Boundary Layer (CBL) flow on the wind turbine wake. The obtained data clearly illustrated that, the wake turbulence intensity is generally higher in CBL than that in the Neutral Boundary Layer (NBL). This is reflected on the velocity distribution in the wake region, such that the wake recovery is faster in CBL as compared with the neutral condition. A similar behavior of the wake flow was also reported during the field measurements of Iungo and Porté-Agel [5]. During these measurements, there was a significant reduction of turbulence activity within the atmospheric surface layer moving from CBL to NBL regimes, which in turn caused the wake to recover faster under convective regime compared with the neutral one.

Chu and Chiang [6] investigated experimentally the effects of the ambient turbulence on the wind turbine wake properties and power performance. The wake velocity deficit was observed to be smaller for high turbulent flow. It was also reported that, the turbulence intensity decreases in the downstream and the largest decay rate was detected in the intermediate region, defined from $2.5 D$ to $4 D$ beyond the turbine.

Hancock and Pascheke [7] carried out wake measurements in the EnFlo stratified-flow wind tunnel operating under NBL and Stable Boundary Layer (SBL) flow. Their measured data demonstrated that, the wake recovery is slower in SBL compared with NBL condition. This behavior is qualitatively consistent with the field measurements reported by Magnusson and Smedman [8].

Besides the field measurements and wind tunnel experiments of wind turbine wakes, analytical and numerical models have also addressed the effect of the inflow conditions on the wind turbine wake behavior. Prospathopoulos et al. [9] investigated numerically the wake characteristics of a $5 \mathrm{MW}$ wind turbine, operating at different inflow turbulence intensities of 5, 13 and 20\%. Faster wake recovery was observed as the ambient turbulence level increased. In addition, the deficit was practically negligible at the distance of $20 D$.

Makridis and Chick [10] used the CFD software FLUENT 12.0 to investigate the wakes of a stand-alone wind turbine, operating in neutral atmospheric wind flow over a complex terrain. The lateral predicted wake deficits were in good comparison with the experimental data at all downstream locations, except at a distance of $4 D$, where a maximum deficit over prediction of $15 \%$ was noticed. On the other hand, the CFD results of the vertical distribution of the wake deficits did not match very well with the measurements.

Recently, Abkar and Porté-Agel [11] used LES frame work combined with the actuator disk model with rotation, proposed by Porté-Agel et al. [12], to investigate the effect of atmospheric thermal stability on the wind turbine wakes of Vestas V80-2MW wind turbine. Their results indicated that, the spatial distribution of the wake characteristics is strongly affected by the different atmospheric stratifications. In particular, the wake recovers faster under convective conditions compared with the other two conditions due to higher turbulence level of incoming wind in CBL. More comprehensive review on the numerical and experimental studies of the 
wind turbine wakes can be viewed in Sanderse et al. [13], Vermeer et al. [14], and Göçmen et al. [15].

The present paper is devoted to testing the different operating conditions of the upstream wind flow on the wake behavior at different ambient stability conditions. Moreover, the amount of the wake deficits and the available wind power in the wake region have been calculated at different downstream locations from the turbine in order to identify the required downstream distance for installing another wind turbine. Abuhegazy et al. [16] tested the standard $k-\varepsilon$ model as well as the two modified models of Crespo et al. [17] and El Kasmi and Masson [18] for the turbulence modulation in studying the wind turbine wakes behavior. It was pointed out from their study that, the modified version of the standard $k-\varepsilon$ model, suggested by El Kasmi and Masson [18], gives satisfactory results as compared with the reported experimental data. Hence, such model is employed in the present simulation.

\section{Mathematical Modeling}

The numerical simulation of the wind turbine wakes under different thermally-stratified conditions of the ABL is based on RANS equations. The flow is considered as steady ideal gas. Also, the buoyancy effect due to atmospheric thermal stratifications is taken into account in the turbulence model equations. The continuity and momentum equations are considered according to AbdelSalam and Ramalingam [19], and solved.

The continuity and momentum equations are given by,

$$
\begin{aligned}
& \frac{\partial \rho u_{j}}{\partial x_{j}}=0 \\
& \frac{\partial \rho u_{i} u_{j}}{\partial x_{j}}=-\frac{\partial p}{\partial x_{i}}+\frac{\partial \tau_{i j}}{\partial x_{j}}
\end{aligned}
$$

where, $\tau_{i j}$ is the Reynolds stress tensor of eddy viscosity model that is given by

$$
\tau_{i j}=\mu_{e}\left(\frac{\partial u_{i}}{\partial x_{j}}+\frac{\partial u_{j}}{\partial x_{i}}\right)
$$

where, $\mu_{e}=\mu+\mu_{t}$ is the effective viscosity, in which $\mu$ is the laminar viscosity and $\mu_{t}$ is the turbulent viscosity, calculated from the adopted turbulence closure.

The turbulence model, suggested by El Kasmi and Masson [18], was modified by Abuhegazy et al. [16] to represent the buoyancy generated turbulence. This modification allows to perform the simulation of the thermally-stratified ABL without the need to solve the energy equation. Such model is adopted for the turbulence modulation with the model equations, reported in Abuhegazy et al. [16].

\section{Computational Domain and Grid Generation}

The computational domain, shown in Fig. 1, is extended $30 D$ in the streamwise direction (xcoordinate), $8 D$ in the lateral direction (y- coordinate) and $6 D$ in the vertical direction (zcoordinate). Using these dimensions, the blockage ratio of the actuator disk is about $1.636 \%$, which is in the acceptable range, reported by Howell et al. [20]. The simulated actuator disk is positioned $5 D$ downstream of the inlet boundary, in the middle distance between the two lateral sides of the domain. 
The three-dimensional CAD model of the computational domain has been completed using SolidWorks program, then it has been imported to ANSYS ICEM program for generating the computational grid. A grid independent study (not presented here) has been performed utilizing unstructured mesh of suitable grid sizes with four different grid resolutions $(0.5,0.8,1.2$ and 1.55 million cells) in the simulations. Figure 2 shows the applied grid of 1.2 million cells. The dimensionless near wall distance $\left(\mathrm{y}^{+}\right)$is found to be in the range of 40-90, which is suitable for the adopted near wall functions.

\section{Boundary Conditions}

The following boundary conditions are considered and applied for the computational domain. The distribution of the flow variables including velocity, turbulent kinetic energy, and dissipation rate are prescribed for SBL $(L>0)$ and CBL $(L<0)$ based on the Monin-Obukhov similarity theory, as reported by Alinot and Masson [21]. At the outlet boundary, the pressure outlet $\mathrm{BC}$ with constant atmospheric pressure value is employed. Each lateral side is far from the center at the turbine of about $4 D$. Thus, both of them are sufficiently far from the wake core to be treated using symmetry $\mathrm{BC}$, in which the normal gradients to the surface for all flow variables across this boundary are neglected. The upper boundary is prescribed at a sufficient vertical height such that the influence from the wake is not significant. As a result, the upstream flow properties at the same height are maintained constant at this boundary. The near wall treatment of Parente et al. [22] is implemented near the ground surface. For the rotor treatment, the actuator disk method is used to represent the effect of the turbine rotor on the flow. The FLUENT fan model [23] is adopted for describing the actuator disk. In this model, an additional sink term is added to the momentum equation through specifying the pressure drop across the disk based on the thrust coefficient. This drop of pressure is calculated from the following equation;

$$
\Delta p=0.5 \rho C_{T} U_{h u b}^{2}
$$

where, $\rho$ is the air density, and $C_{T}$ is the turbine thrust coefficient that is obtained from the thrust coefficient curves against wind velocity for the modeled turbines at the given hub height velocity $U_{h u b}$.

\section{Solution Procedure}

Once the mesh file is created by the ANSYS ICEM program, it is exported to ANSYS FLUENT 15 solver, by which the complete set of the governing equations are solved. The control volumebased technique is used for the discretization. Moreover, The Semi-Implicit Method for Pressure-Linked Equations (SIMPLE) algorithm [24] is used for the pressure-velocity coupling and the first-order upwind scheme is used for all dependent variables. The inlet, wall, outlet boundary conditions and the additional source terms, added to the governing equations, are implemented to the solver using User Defined-Functions (UDF), programed in C - language. The program has been run, until the order of the convergence residuals reach at least $10^{-5}$ for the solved equations.

\section{Results and Discussion}

In this section, the effects of the inflow turbulence intensity $\left(T I=\sqrt{2 k / 3} / U_{h u b}\right)$, and wind speed on the wind turbine wakes behavior are presented and discussed. Furthermore, the amount of the wake deficits, as well as the available wind power in the wake region at different downstream positions from the turbine have been specified for the different operating conditions. The CFD computations are performed on the ECN turbine [25]. Such turbine has 
been selected due to the availability of its thrust coefficient curve against the different wind speeds [26]. Thus, the simulations can be performed for this turbine at different wind speeds.

\subsection{Effect of the Upstream Flow Turbulence Intensity}

Due to the change in the meteorological conditions as well as the various shapes of the ground terrain roughness, the inflow atmospheric wind shears and turbulence intensity vary, even for the same atmospheric stability regime [9]. The influence of the turbulence intensity upstream of the turbine has been studied, by changing its value at the turbine hub height ranging from 6 $\%$ up to $15 \%$ for a fixed hub height velocity of $9.15 \mathrm{~m} / \mathrm{s}$. Further, this analysis has been performed for the SBL condition at $L=200 \mathrm{~m}$ as well as for the CBL condition at $L=-200 \mathrm{~m}$, where $L$ is the Monin-Obukhov length.

\subsubsection{SBL Condition $(L=200 \mathrm{~m})$}

Figures 3 presents the contours of the streamwise velocity through a horizontal plane at the turbine hub height. Such contours are displayed at the SBL case for various inflow turbulence intensities of $6 \%, 8 \%, 10 \%, 12.5 \%$ and $15 \%$, prevailing at the hub height. It is seen that, the turbulence of the main upstream flow has a significant effect on the flow properties of the wind turbine wake. In particular, as the TI increases, the wake recovers faster and hence, the strength and extension of the wake region downstream the turbine decreases. This is a direct consequence of the higher mixing process between the wake flow and the surrounding atmospheric flow, caused by the elevated wind shears of the higher turbulence levels.

In order to provide a more valuable and useful wake representation, the average wake deficit, as well as the available wind power over an equivalent rotor area are calculated in the wake region. The locations downstream of the turbine between $1 D$ and $15 D$ with a step value of $1 D$ are considered for such calculations. Figure 4 presents the percentage change in the wake deficit $\left(U_{d}\right)$ with the normalized downstream distance $(x / D)$ for the different inflow turbulence intensities. The $U_{d}$ is defined as $\left(U_{i n}-U_{w}\right) / U_{i n}$, where $U_{i n}$ and $U_{w}$ are the area weighted average of the axial flow velocity ( $\frac{1}{A} \int u d A$ ), prevailing at the offset surfaces from the turbine rotor with its equivalent area, at inlet and at different downstream locations, respectively, as shown in Fig. 5.

It has been seen from Fig. 3 that, the increase in the TI causes a significant increase in the mixing process between the atmosphere and the wake, which in turn enhances the velocity recovery in the wake. The obtained results of the $U_{d}$ in Fig. 4 ensures this, in which, the wake deficit decreases with the increasing of $T I$, ranging from $39.2 \%$ at $T I=6 \%$ to $28.8 \%$ at $T I=$ $15 \%$ in the near wake region $(x / D=1)$ and from $14 \%$ at $T I=6 \%$ to $2.9 \%$ at $T I=15 \%$ in the very far wake region $(x / D=15)$. Moreover, the rate of decrement of the $U_{d}$ with the streamwise distance for the different turbulence intensities is approximately the same. However, it is higher in the near wake region up to 4-5D than the further downstream distances, for each turbulence level. This is due to the superposition of the highly generated turbulent flow in the near wake region with the ambient turbulence, while the atmospheric turbulence is the only dominant turbulence factor beyond the near wake region.

Figure 6 demonstrates the streamwise distribution of the wind power in the wake region $\left(P_{w}\right)$, normalized by the available inflow wind power at inlet $\left(P_{i n}\right) . P_{i n}$ and $P_{w}$ are calculated as the area integral $\left(\int 0.5 \rho u^{3} d A\right)$ over the same surfaces, used to calculate the percentage wake deficit. It can be depicted from this figure that, the available wind power in the wake region increases with downstream distance and it also increases with increasing the upstream flow turbulence intensity. This is a direct effect of the above-discussed wake deficit, which is the only changeable parameter in the calculation of the wind power. Thus, the wind power in the wake region can be considered to behave as inversely proportional to the wake deficit. Further, the rate of the wind power recovery is higher for the higher turbulence levels, especially up to 
$x / D=5$ when comparing the slope of the $6 \%$ turbulence level with that of the $15 \%$ turbulence level. It is worth noting that, the effect of the turbulence intensity is still significant in the far wake region. The wind power available in the wake at $x / D=15$ is about $64 \%$ of the inflow wind power for $T I=6 \%$, while it is about $92 \%$ of the inflow wind power for $T I=15 \%$.

\subsubsection{CBL Condition $(L=-200 \mathrm{~m})$}

The contours of the streamwise velocity for the CBL case are presented in Fig. 7 at the same horizontal plane and inflow turbulence intensities, used for the SBL case. It can be concluded from these figures that, the different turbulence intensities influence the wake development in a similar manner to that, discussed above for the SBL flow. However, the major difference of the wake characteristics between the SBL flow and the CBL one is the value of the TI, which in turn affects the wake characteristics.

Moreover, Figs. 8 and 9 show the distributions of the $U_{d}$ and the fractional wind power in the wake region at the considered turbulence intensities. Similar behavior to the SBL condition is also noticed here for the CBL one. High turbulence intensities enable the entrainment of the main flow into the wake as well as to recover the velocity in the wake quickly. It should be noted that, the value of the $U_{d}$ is smaller in the CBL case for each turbulence level compared with its equivalent value at the same TI in the SBL case, see Figs. 4 and 8. This difference in the $U_{d}$ values increases as the $T I$ increases, particularly in the near wake region.

As a consequence to the $U_{d}$ illustration, the available wind power is higher in the CBL than the SBL condition for the same value of the turbulence intensity, see Figs. 6 and 9. Moreover, the rate of the wind power recovery in the CBL is higher than in the SBL flow and this recovery rate increases as the $T I$ increases in the near wake region up to $x / D=5$.

\subsection{Effect of the Hub Height Velocity}

The incoming wind speed is expected to be a critical parameter that affects the wind turbine wake characteristics. Hence, the effect of the $U_{h u b}$ has been tested for the SBL $(L=200 \mathrm{~m})$ as well as the CBL $(L=-200 m)$ conditions, used in the previous section.

For such purpose, $U_{h u b}$ has been varied from $5 \mathrm{~m} / \mathrm{s}$ to $15 \mathrm{~m} / \mathrm{s}$ considering the corresponding value of the $C_{T}$ for each velocity [26].

\subsubsection{SBL Condition $(L=200 \mathrm{~m})$}

The variation of the $U_{d}$ with the streamwise direction in the wake region for the different hub height velocities at the SBL regime is shown in Fig. 10. It can be depicted that, the wake develops and recovers as it moves away from the turbine and hence, $U_{d}$ decreases for any of the tested inflow wind velocities. This rate of the reduction in $U_{d}$ is more dramatic at the low wind speeds of $5 \mathrm{~m} / \mathrm{s}$ and $7 \mathrm{~m} / \mathrm{s}$ than the higher velocity values of $12 \mathrm{~m} / \mathrm{s}$ and $15 \mathrm{~m} / \mathrm{s}$, especially in the near wake region. Moreover, $U_{d}$ values immediately downstream the turbine at $x / D=1$ are higher for the lower inflow wind speeds, as previously reported by Abdelsalam et al. [27] for the NBL flow condition. This is resulted from the basic idea of the turbine thrust, in which the resistance to the flow by the turbine increases as the $C_{T}$ increases. Accordingly, for the lower inflow wind speeds (i.e., higher $C_{T}$ values), the higher wake deficits are obtained. It is also noted that, the wake flow at $U_{h u b}=5 \mathrm{~m} / \mathrm{s}$ and $7 \mathrm{~m} / \mathrm{s}$ follows the same attitude and has nearly the same $U_{d}$ at $x / D=1$, as the $C_{T}$ values of these two wind speeds $(0.831$ and 0.826 , respectively) are very close to each other.

A comparison of the fractional wind power distribution in the wake region at the tested hub height velocities is presented in Fig. 11. As previously pointed out, the wind power in the wake region is inversely proportional to the wake deficit. Therefore, the fractional wake wind power is found to increase with the wake expansion downstream of the turbine. It also increases as $U_{h u b}$ increases. The wind power increases more drastic compared to the $U_{d}$, because of its cubic 
relation with the wind speed. In the near wake region up to $x / D=3$, the power in the wind flow is around $20 \%$ of the total available wind power in the upstream flow at $U_{h u b}=5 \mathrm{~m} / \mathrm{s}$, while such value is approximately $71 \%$ at $U_{h u b}=15 \mathrm{~m} / \mathrm{s}$.

\subsubsection{SBL Condition $(L=200 \mathrm{~m})$}

In the case of the CBL regime, Figs. 12 and 13 show the change of the $U_{d}$ and the fractional wind power in the wake region for the various investigated hub height velocities. Comparable observations to the SBL condition are also achieved for the CBL regime, accompanied with slightly smaller values of the $U_{d}$ in the near wake region at the CBL, case as compared with the SBL one. This emphasizes the difference in the wake structure (i.e., the wake recovery rate) between both the SBL and CBL flow conditions.

\section{Conclusions}

In the current study, the influence of the different inflow atmospheric conditions, including the inflow turbulence intensity and wind speed, on the wind turbine wakes has been numerically investigated. The percentage wake deficits, as well as the available wind power in the wake region at different downstream locations from the turbine have been calculated for the different operating conditions. A modified $k-\varepsilon$ model has been employed. The buoyancy effect of the SBL and CBL is artificially included, without solving the energy equation. The obtained results indicated that, the increase in the turbulence intensity causes a significant increase in the mixing process between the atmosphere and the wake. As a result, the rate of the wind power recovery is higher for the higher turbulence levels, especially in the near wake region up to $x / D=5$. Additionally, for the lower inflow wind speeds, higher wake deficits are observed. The rate of the reduction in the wake deficit, as proceeded downstream, is more dramatic at the low wind speeds than the higher speeds, especially in the near wake region. It is also found that, though the extension of the wake is perceptible until $15 D$ downstream the turbine, the positions more than $7 D$ can be considered suitable for implementing another wind turbine. In such case, the wind power is close to $69.5 \%$ for the turbines' rated wind speed of $12 \mathrm{~m} / \mathrm{s}$, and $86.8 \%$ for the rated wind speed of $15 \mathrm{~m} / \mathrm{s}$.

\section{References}

[1] L. P. Chamorro and F. Porté-Agel, "Effects of Thermal Stability and Incoming Boundary-Layer Flow Characteristics on Wind-Turbine Wakes: A Wind-Tunnel Study," Boundary-Layer Meteorol., vol. 136, no. 3, pp. 515-533, 2010.

[2] L. P. Chamorro and F. Porté-Agel, "A wind-tunnel investigation of wind-turbine wakes: Boundary-Layer turbulence effects," Boundary-Layer Meteorol., vol. 132, no. 1, pp. 129-149, 2009.

[3] T. Maeda, Y. Kamada, J. Murata, S. Yonekura, T. Ito, A. Okawa, and T. Kogaki, "Wind tunnel study on wind and turbulence intensity profiles in wind turbine wake," J. Therm. Sci., vol. 20, no. 2, pp. 127-132, 2011.

[4] W. Zhang, C. D. Markfort, and F. Porté-Agel, "Wind-Turbine Wakes in a Convective Boundary Layer: A Wind-Tunnel Study," Boundary-Layer Meteorol., vol. 146, no. 2, pp. 161-179, 2013.

[5] G. V. Iungo and F. Porté-Agel, "Volumetric lidar scanning of wind turbine wakes under convective and neutral atmospheric stability regimes," J. Atmos. Ocean. Technol., vol. 31, no. 10, pp. 2035-2048, 2014.

[6] C. R. Chu and P. H. Chiang, "Turbulence effects on the wake flow and power production of a horizontal-axis wind turbine," J. Wind Eng. Ind. Aerodyn., vol. 124, pp. 82-89, 2014. 
[7] P. E. Hancock and F. Pascheke, "Wind-Tunnel Simulation of the Wake of a Large Wind Turbine in a Stable Boundary Layer: Part 2, the Wake Flow," Boundary-Layer Meteorol., vol. 151, no. 1, pp. 23-37, 2014.

[8] M. Magnusson and A. S. Smedman, "Influence of Atmospheric Stability on Wind Turbine Wakes," Wind Eng., vol. 18, no. 3, pp. 139-152, 1994.

[9] J. Prospathopoulos, E. S. Politis, and P. K. Chaviaropoulos, "Modelling wind turbine wakes in complex terrain," in Proceedings of the EWEC, Brussels, Belgium, 2008.

[10] A. Makridis and J. Chick, "Validation of a CFD model of wind turbine wakes with terrain effects," J. Wind Eng. Ind. Aerodyn., vol. 123, pp. 12-29, 2013.

[11] M. Abkar and F. Porté-Agel, "Influence of atmospheric stability on wind-turbine wakes: A large-eddy simulation study," Phys. Fluids, vol. 27, no. 3, 035104, 2015.

[12] F. Porté-Agel, Y. T. Wu, H. Lu, and R. J. Conzemius, "Large-eddy simulation of atmospheric boundary layer flow through wind turbines and wind farms," J. Wind Eng. Ind. Aerodyn., vol. 99, no. 4, pp. 154-168, 2011.

[13] B. Sanderse, S. P. van der Pijl, and B. Koren, "Review of CFD for Wind-Turbine Wake Aerodynamics," Wind Energy, vol. 14, no. 7, pp. 799-819, 2011.

[14] L. J. Vermeer, J. N. Sørensen, and A. Crespo, "Wind turbine wake aerodynamics," Prog. Aerosp. Sci., vol. 39, no. 6, pp. 467-510, 2003.

[15] T. Göçmen, P. Van Der Laan, P. Réthoré, and A. P. Diaz, "Wind turbine wake models developed at the technical university of Denmark : A review," Renew. Sustain. Energy Rev., vol. 60, pp. 752-769, 2016.

[16] M. R. Abuhegazy, A. M. Abdelsalam, I. M. Sakr, and W. A. El-Askary, "Numerical Study on Wind Turbine Wakes under Thermally-Stratified Conditions," in Proceedings of the 17th Int. AMME Conference, pp. 241-257, 19-21 April, 2016.

[17] A. Crespo, F. Manuel, D. Moreno, E. Fraga, and J. Hernandez, "Numerical analysis of wind turbine wakes," in Proceedings of Delphi Workshop on Wind Energy Applications, Delphi, Greece, pp. 15-25, 1985.

[18] A. El Kasmi and C. Masson, "An extended k- $\varepsilon$ model for turbulent flow through horizontal-axis wind turbines," J. Wind Eng. Ind. Aerodyn., vol. 96, no. 1, pp. 103-122, 2008.

[19] A. M. AbdelSalam and V. Ramalingam, "Wake prediction of horizontal-axis wind turbine using full-rotor modeling," J. Wind Eng. Ind. Aerodyn., vol. 124, pp. 7-19, 2014.

[20] R. Howell, N. Qin, J. Edwards, and N. Durrani, "Wind tunnel and numerical study of a small vertical axis wind turbine," Renew. Energy, vol. 35, no. 2, pp. 412-422, 2010.

[21] C. Alinot and C. Masson, " $k-\varepsilon$ Model for the Atmospheric Boundary Layer Under Various Thermal Stratifications," J. Sol. Energy Eng., vol. 127, no. 4, pp. 438-443, 2005.

[22] A. Parente, C. Gorlé, J. van Beeck, and C. Benocci, "Improved k- $\varepsilon$ model and wall function formulation for the RANS simulation of ABL flows," J. Wind Eng. Ind. Aerodyn., vol. 99, pp. 267-278, 2011.

[23] “ANSYS FLUENT 15 Theory Guide," Fluent Inc., Lebanon.

[24] S. V. Patankar, Numerical heat transfer and fluid flow. McGraw-Hill, New York, 1980.

[25] L. A. H. Machielse, P. J. Eecen, H. Korterink, S. . P. van der Pijl, and J. G. Schepers, "ECN test farm measurements for validation of wake models," in Proceedings of the EWEC, Milan, vol. 7, pp. 5-16, 2007.

[26] E. T. G. Bot, G. P. Corten, and P. Schaak, "FluxFarm A Program to Determine Energy Yield of Wind Turbines in a Wind Farm," Technical Report ECN-C-06-029, 2006.

[27] A. M. Abdelsalam, K. Boopathi, S. Gomathinayagam, S. S. Hari Krishnan Kumar, and V. Ramalingam, "Experimental and numerical studies on the wake behavior of a horizontal axis wind turbine," J. Wind Eng. Ind. Aerodyn., vol. 128, pp. 54-65, 2014. 


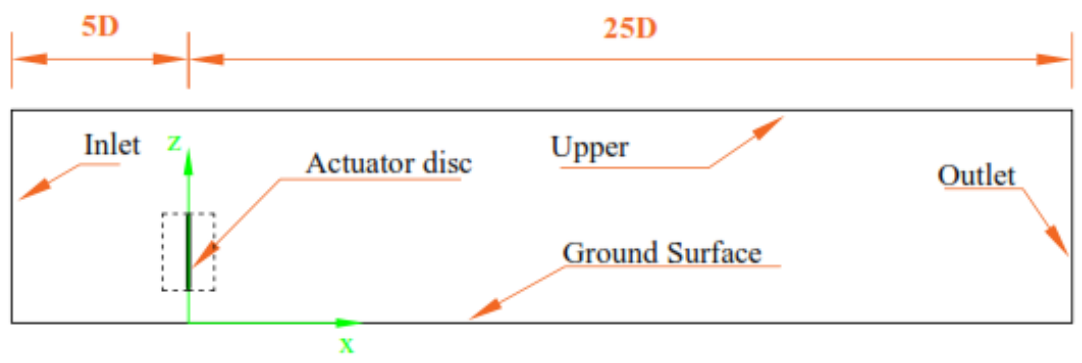

Front View

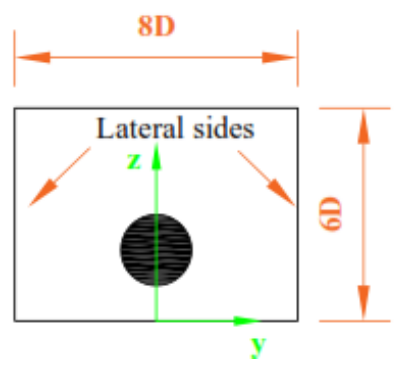

Side View

Fig. 1. Schematic representation of the computational domain (Not to scale)

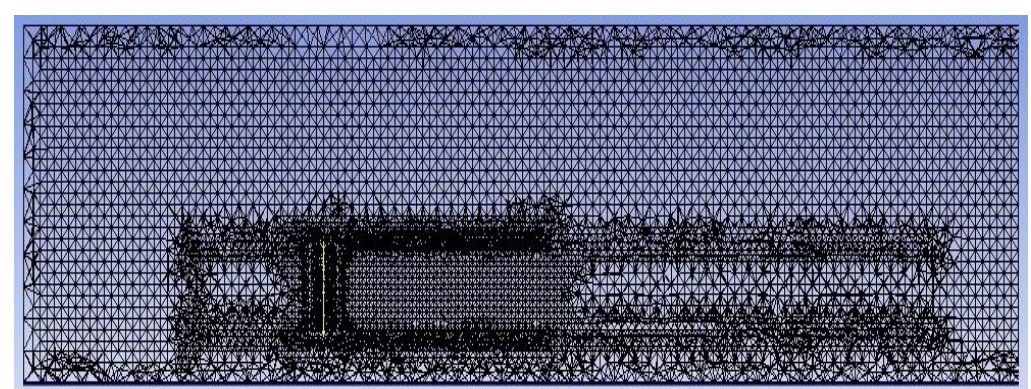

Front view

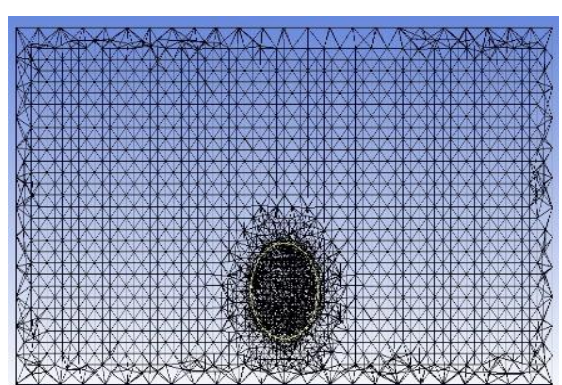

Side view

Fig. 2. The computational grid at a vertical center plane across the actuator disk 


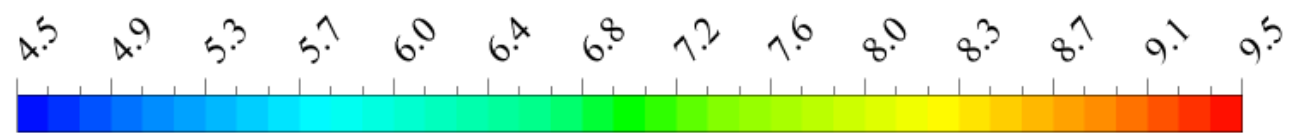

Streamwise velocity

$\mathrm{m} / \mathrm{s}$
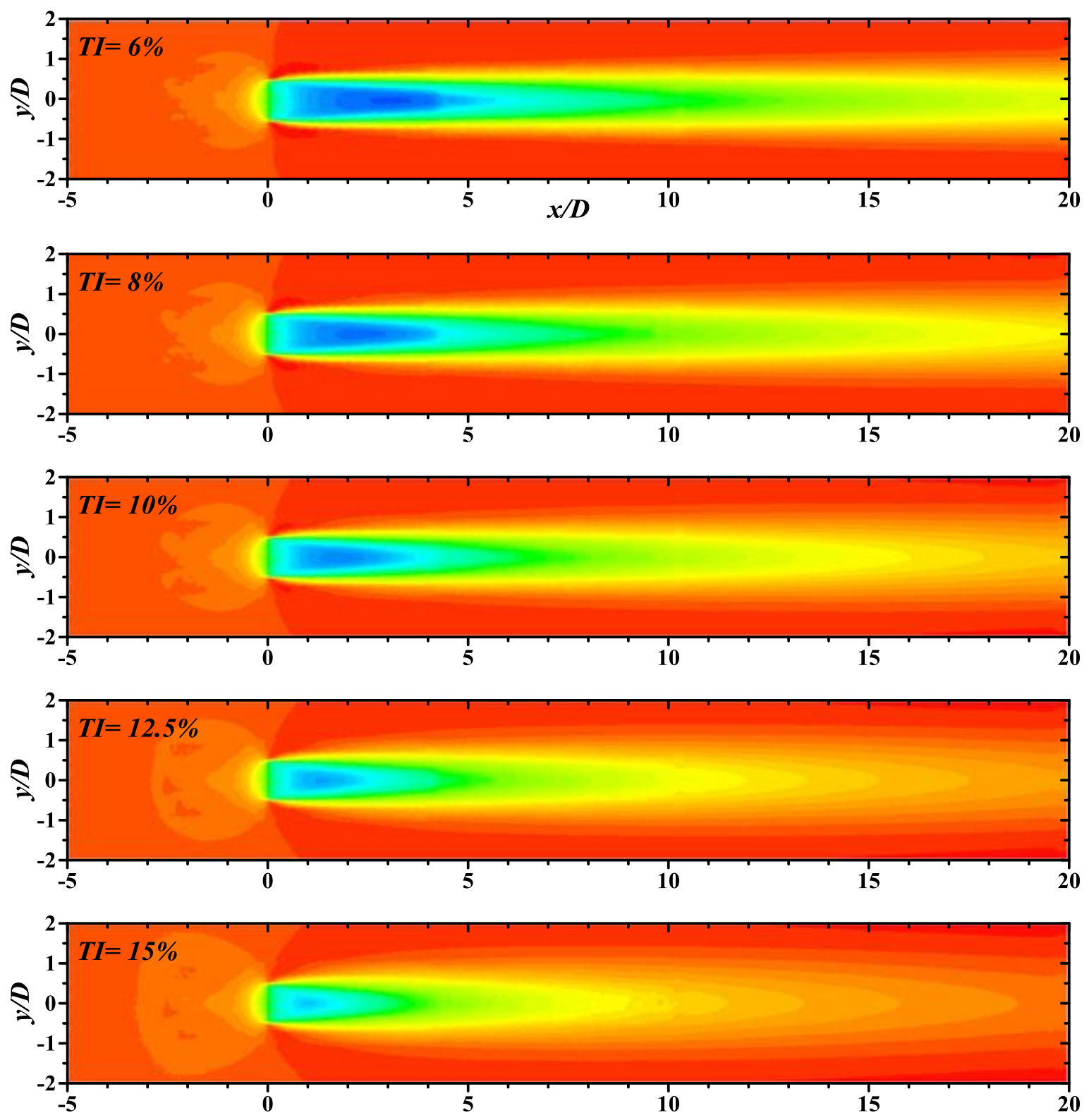

Fig. 3. Contours of the streamwise velocity in a horizontal plane at the hub height at the different turbulence intensities, for the SBL condition $(L=200 \mathrm{~m})$ 


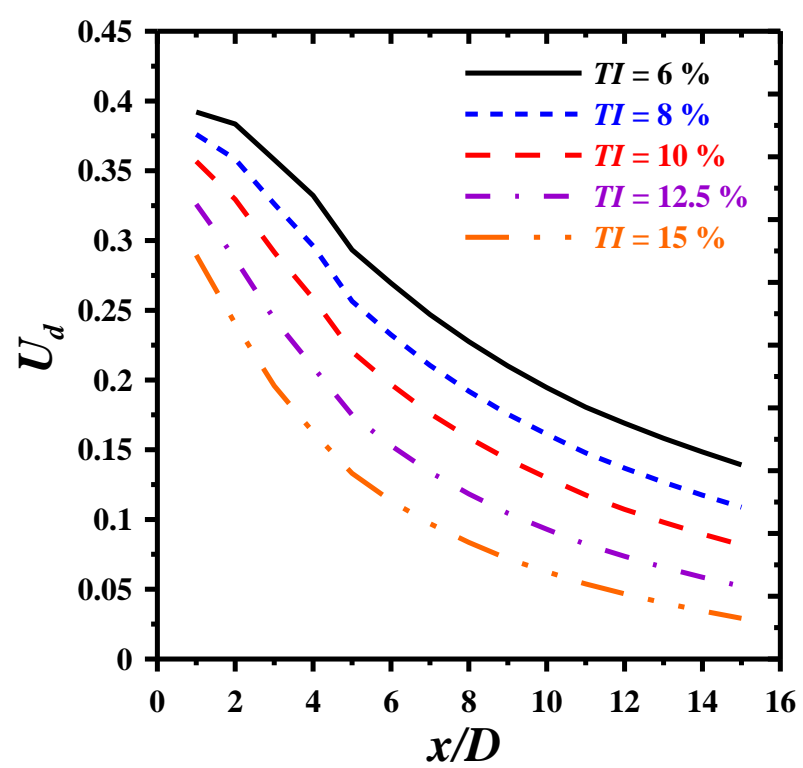

Fig. 4. Percentage velocity deficit $\left(U_{d}\right)$ in the wake region at the different turbulence intensities, for the SBL condition $(L=200 \mathrm{~m})$

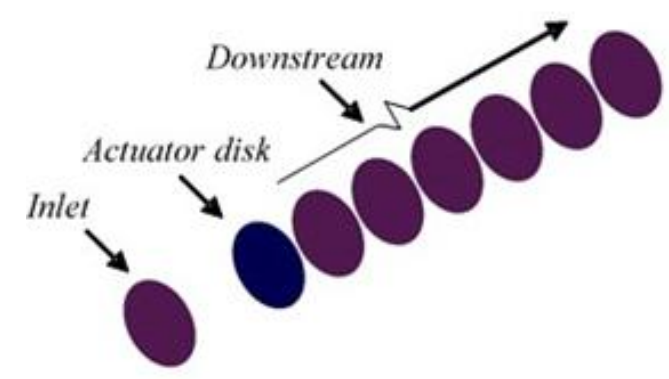

Fig. 5. Offset surfaces, used in the calculation of the area integrals

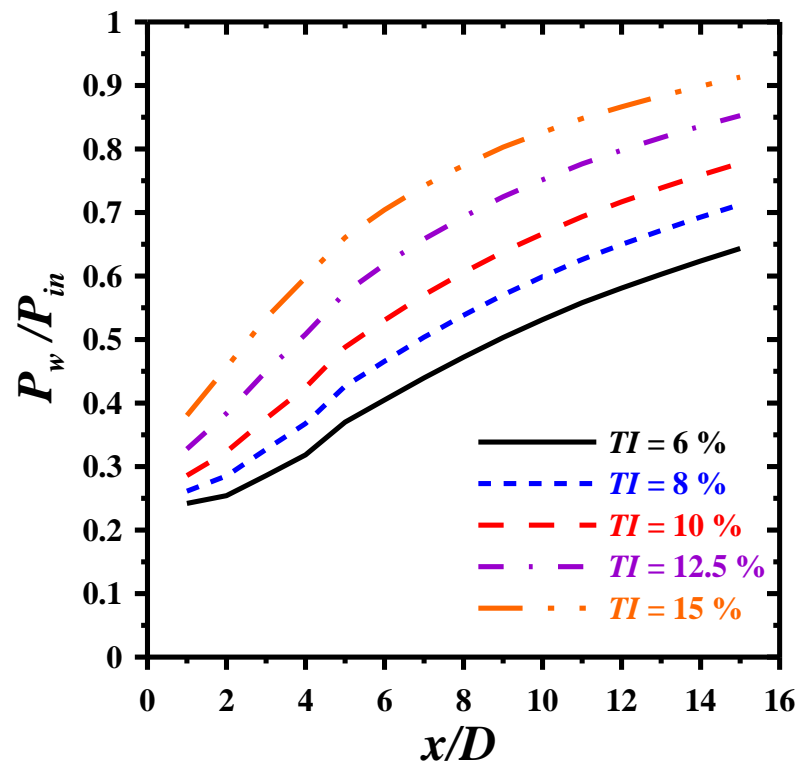

Fig. 6. The fractional wind power in the wake region at the different turbulence intensities, for the SBL condition $(L=200 \mathrm{~m})$ 


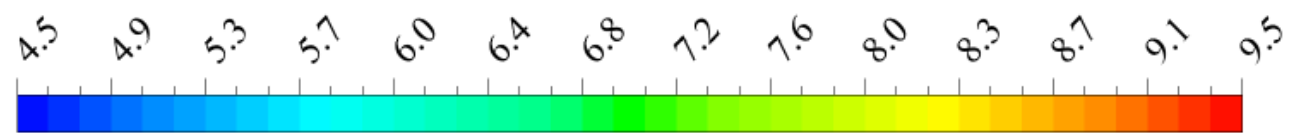

Streamwise velocity

$\mathrm{m} / \mathrm{s}$
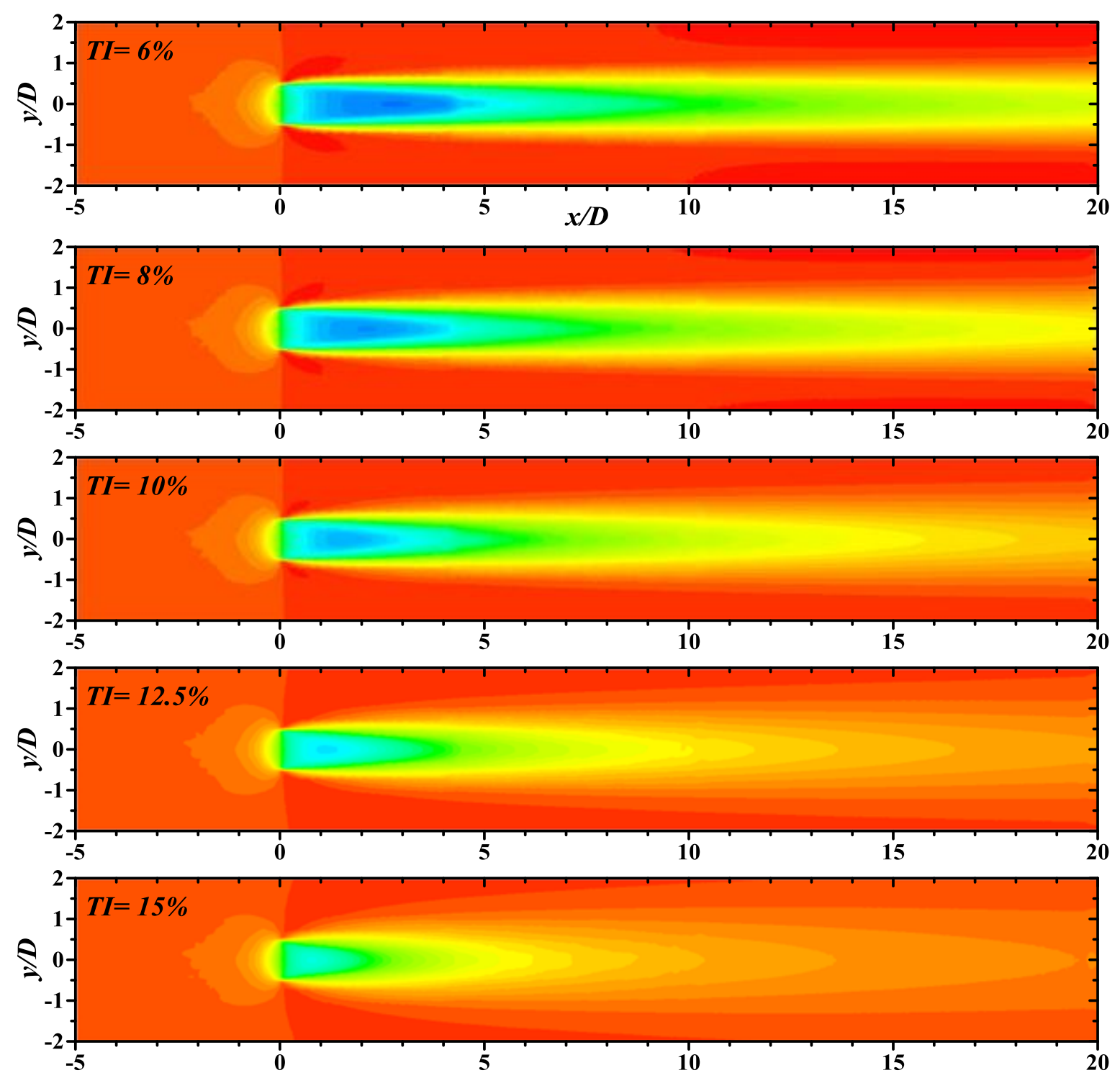

Fig. 7. Contours of the streamwise velocity in a horizontal plane at the hub height at the different turbulence intensities, for the CBL condition $(L=-\mathbf{2 0 0} \mathrm{m})$ 


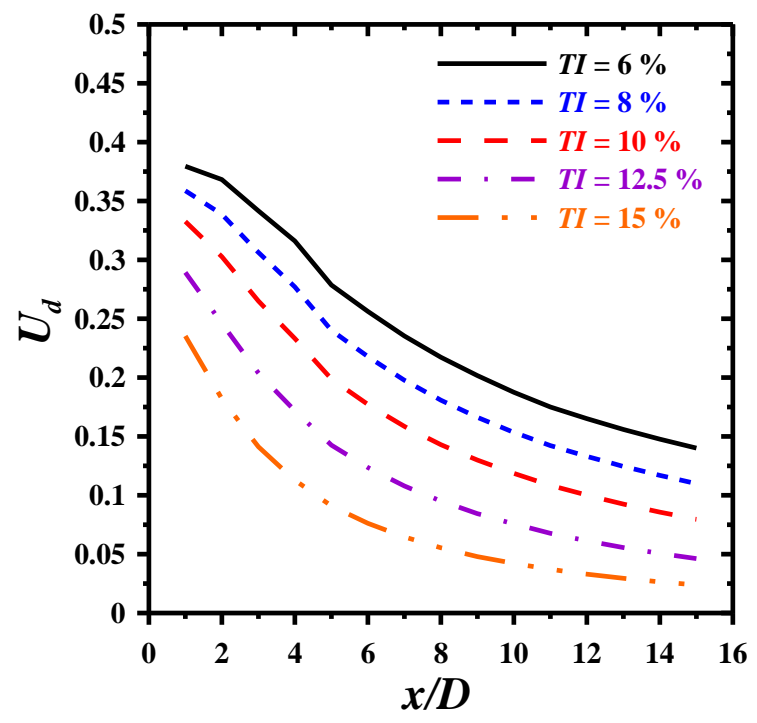

Fig. 8. Percentage velocity deficit $\left(U_{d}\right)$ in the wake region at the different turbulence intensities, for the CBL condition $(L=-200 \mathrm{~m})$

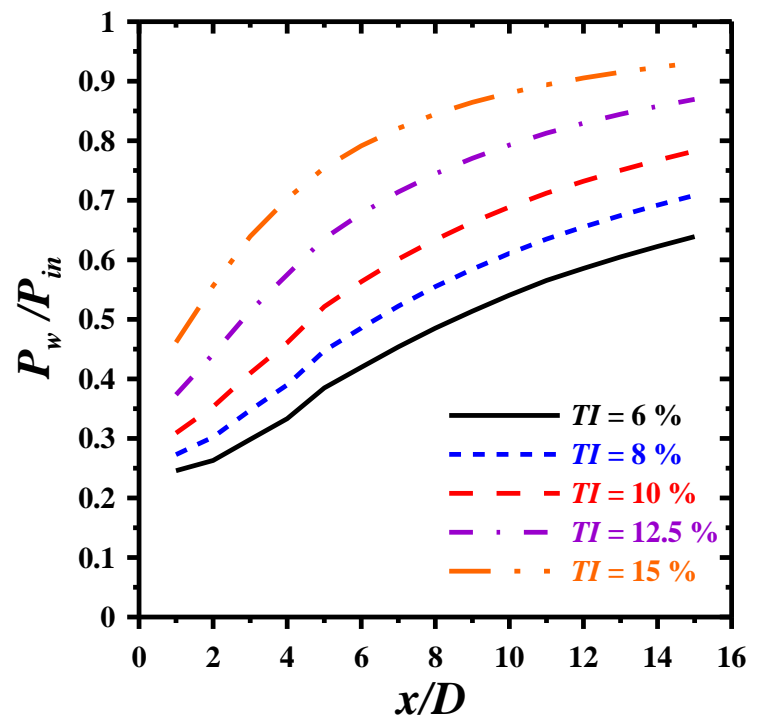

Fig. 9. The fractional wind power in the wake region at the different

turbulence intensities, for the CBL condition $(L=-200 \mathrm{~m})$

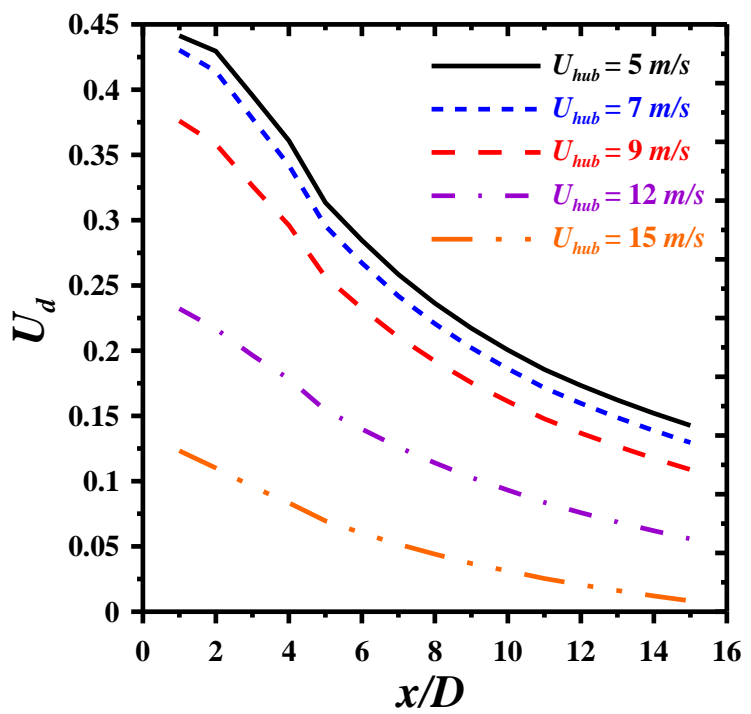

Fig. 10. Percentage velocity deficit $\left(U_{d}\right)$ in the wake region at the different hub height velocities, for the SBL condition $(L=200 \mathrm{~m})$ 


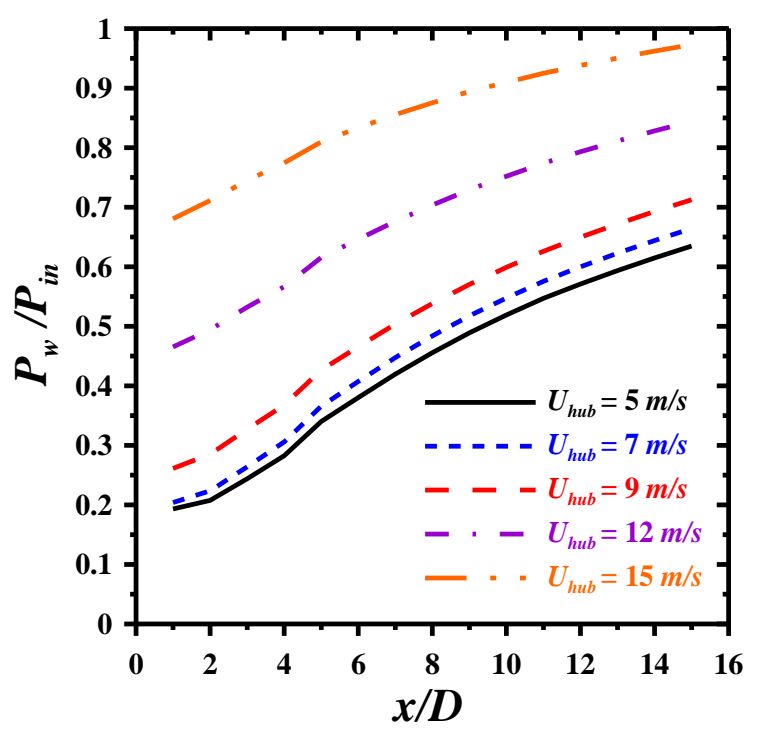

Fig. 11. The fractional wind power in the wake region at the different hub height velocities, for the SBL condition $(L=200 \mathrm{~m})$

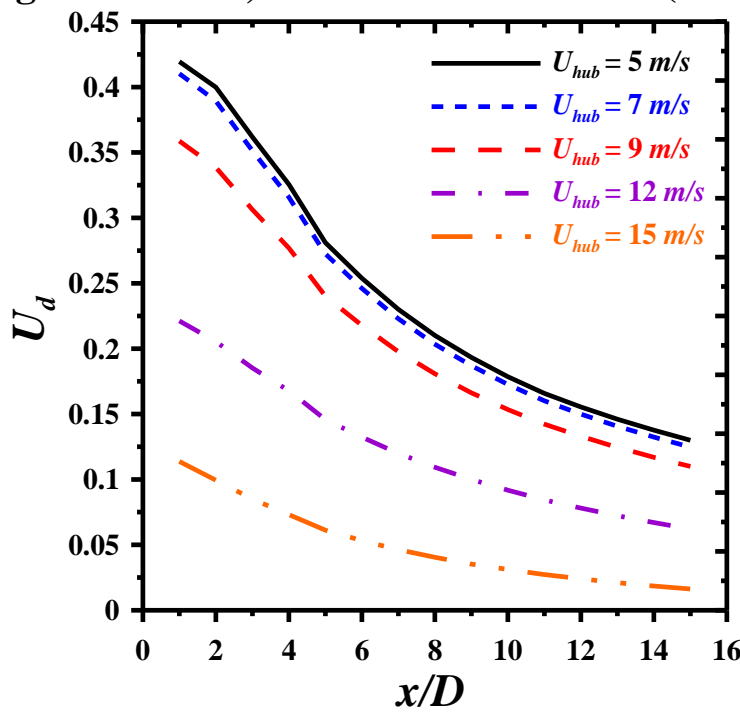

Fig. 12. Percentage velocity deficit $\left(U_{d}\right)$ in the wake region at the different hub height velocities, for the CBL condition $(L=-200 \mathrm{~m})$

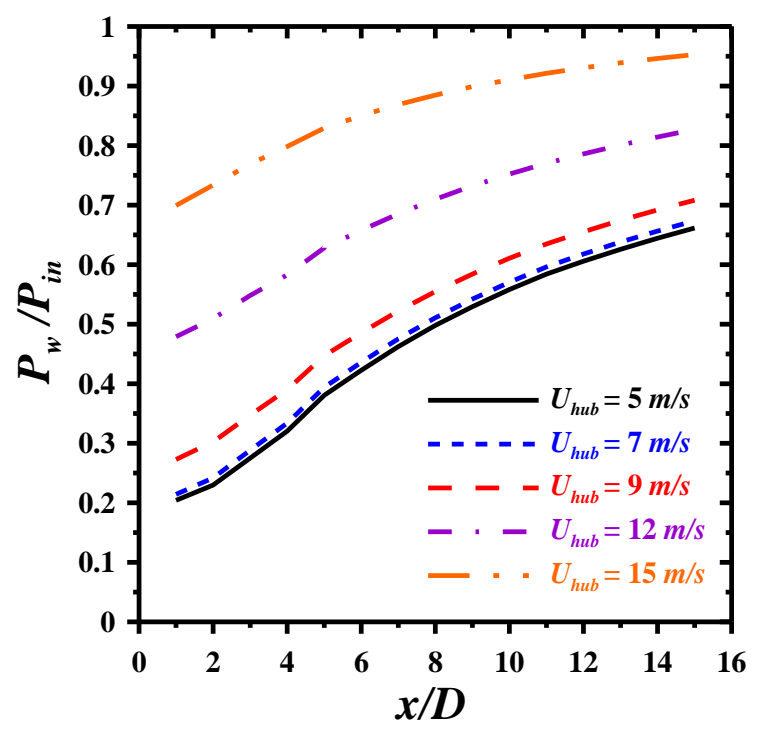

Fig. 13. The fractional wind power in the wake region at the different hub height velocities, for the $\mathrm{CBL}$ condition $(L=-200 \mathrm{~m})$ 